\title{
Paper
}

CL-mWSNs: Cross Layer

\section{Model-Based QoS Centric Routing Protocol for Mission-Critical Cooperative Communication in Mobile WSNs}

\author{
Kummathi Chenna Reddy ${ }^{1}$, Geetha D. Devanagavi ${ }^{1}$, and Thippeswamy M. N. ${ }^{2}$ \\ ${ }^{1}$ School of Electronics \& Communication, REVA University, Bangalore, India \\ ${ }^{2}$ Department of Computer Science, Nitte Meenakshi Institute of Technology, Bangalore, India
}

https://doi.org/10.26636/jtit.2019.129318

\begin{abstract}
The paper presents a robust QoS centric routing protocol for mission-critical communication over mobile Wireless Sensor Networks (CL-mWSN) that exploits dynamic network states from the different layers of the IEEE 802.15.4 protocol stack to make the routing decision. The CL-mWSN protocol exploits three key layers: application layer, network layer and MAC layer. It exhibits proactive network and node table management, service differentiation, fair resource scheduling and congestion detection, avoidance at the network layer, as well as dynamic link quality estimation and packet injection rate estimation at the MAC layer to assess its candidature as the best forwarding node for QoS-centric mission-critical communication. Simulation reveals that the proposed routing model exhibits higher throughput, minimum loss and deadline miss ratio that augments QoS provision in mobile WSNs.
\end{abstract}

Keywords-cross-layer signaling, link quality estimation, quality of service, wireless sensor networks.

\section{Introduction}

Over the past few years, the development of wireless communication technologies has demanded mobile Wireless Sensor Networks (WSNs) to serve numerous applications in which assuring QoS-centric and reliable communication is a must. The application-specific demands and customer preferences have turned Quality of Service (QoS) demands into the decisive selection criterion. Such demands have motivated the academia sector to design a more effective and cost-efficient communication system, with WSNs being considered a broad research domain.

Unlike classic WSN systems with static network deployment, mobile WSNs received more attention in terms of their further optimization. Relevant augmentation could turn mobile WSNs into a low-cost and efficient alternative for the classic ad-hoc networks or mobile ad-hoc networks (MANETs). Mobile WSNs may rely on random deployment of nodes across the network, enabling the nodes to communicate in an ad-hoc manner. However, such node deployment and mobility patterns might impose variations in topology, network states and node characteristics, such as congestion, buffer unavailability, data drop, link outage, etc. These adverse effects could cause a deterioration in QoS and unreliability of communication. In mobile WSNs, some or even all nodes may function as a router to support communication between two hosts in typical communication environments. This may be achieved by employing multi-hop transmissions. Noticeably, being a decentralized network solution, the inclusion of mobility in mobile WSNs could make network management highly complicated and, hence, could adversely affect the satisfaction of required QoS levels.

In mobile WSNs, each node relies on a routing model to perform communication with neighboring nodes, or forwards data to the next hop towards the destination. If two sensor nodes are within radio range, they may communicate directly. Otherwise, multi-hop transmission is used to forward data to the next hop node to ensure that it is reliably received at the destination. In static WSNs, routing may be performed through reactive node management, while in mobile WSNs classic reactive routing cannot be applied due to network parameter changes, meaning that a well-defined proactive network management approach and good network awareness are required. In mobile WSNs, the selection of the best forwarding node (BFN) plays a vital role.

With the aforementioned motivations taken into consideration, the emphasis of this research paper is placed on developing a novel and robust BFN for communication purposes. In WSNs, the communicated data may be of two types: real-time data (RTD) and non-real-time (NRT) data, where the delivery of RTD, which commonly has the form of event-driven critical data, often dominates the prioritization process to assure timely data delivery at the destination. On the other hand, NRT data may also be of significance for meeting user demands. QoS-centric resource provision to RTD while ensuring the maximum possible availability of resources for NRT may be of utmost significance for maintaining the optimized trade-off between these two types of data communication. In such cases, identification 
of RTD among the data sequence containing NRT may play a decisive role in QoS-centric resource allocation and prioritization.

To meet this demand, various service differentiation (SD) approaches may be applied. The use of a well-planned SD scheme can assure both RTD as well as NRT data classification that, in turn, may help in optimal resource allocation to meet QoS needs. This may help the MAC and network layers understand the nature of the data and perform optimal resource scheduling. For the provision of QoS, the other key demand is timely data delivery, where selecting a node with the shortest holding period or considering packet velocity may be suitable for BFN. In addition, this may assist the PHY layer in performing dynamic power management (DPM) and link-adaptive transmission scheduling [1].

The other shortcomings of mobility include congestion and link vulnerability. Hence, assessing these two parameters at the node level can help MAC select only the node with optimum parameters (congestion-free and higher link quality) to make the transmission decision. Therefore, retrieving these key parameters (link quality, congestion, resource availability, packet velocity, etc.) and sharing them across the layers of the protocol stack may ensure optimal BFN selection to guarantee the provision of QoS over mobile WSNs. In practice, these dynamic network parameters can be estimated at the different layers of the protocol stack, and can be shared, at a later phase, with other layers to make the optimized routing decision. The use of a cross-layer network design (CND) may be a novel solution enabling to achieve the desired results.

In this paper, a robust and efficient routing protocol named "QoS-centric routing protocol for mission-critical communication over mobile WSNs (CL-mWSN)" has been developed. As a QoS-centric solution, our proposed CL-mWSN intends to achieve high packet delivery ratio (PDR), higher throughput, minimum packet drop, low latency and endto-end delay, as well as maximum possible resource (i.e. bandwidth) utilization [2]. The proposed CL-mWSN can be stated as a geographical forwarding routing protocol, as it exploits dynamic network parameters to make the routing decision. To ensure network awareness, CL-mWSN applies the proactive network management approach, where node parameters are estimated dynamically and updated proactively to make optimized BFN selection. Unlike classic routing protocols, where single parameters - such as residual energy, link quality, signal to noise ratio (SNR), etc. - are used to perform BFN selection, CL-mWSN applies multiple parameters obtained from the different layers of the protocol stack for routing-related tasks. Here, CL-mWSN exploits the following key parameters: buffer capacity, packet velocity, link quality, distance, etc. to perform BFN selection, in order to ensure reliable data transmission with a minimum probability of data drop (due to the minimum or negligible probability of link outage), and a minimum deadline miss ratio (DMR). CL-mWSN intends to develop a highly robust resource scheduling scheme that could ensure optimal resource provision to event-driven
RTD data, while ensuring that a maximum amount of resources is available to NRT data. Undeniably, it may play a significant role in managing the optimized QoS tradeoff for both RTD and NRT data. The CL-mWSN protocol incorporates enhanced service differentiation and fair resource scheduling, proactive network management, congestion detection and routing decision model at the link layer, dynamic link quality and packet injection rate estimation at the MAC layer, and power switching at the PHY layer of the IEEE 802.15.4 protocol stack. The overall routing has been developed using the Matlab simulation platform, where the simulation output has exhibited higher PDR, packet loss ratio (PLR) and DMR results compared to other state-of-art technologies.

\section{Table 1}

List of used abbreviations

\begin{tabular}{|c|c|}
\hline Variable & Description \\
\hline $\mathbb{N}_{\text {Table }}$ & Proactive node table \\
\hline$B F N i$ & Best forwarding node \\
\hline $\mathbb{N}_{j}$ & Number of nodes (one hop distant nodes) \\
\hline $\mathrm{Eucl}_{d}$ & $\begin{array}{l}\text { Euclidean distance in between the best } \\
\text { forwarding node to the nearest destination }\end{array}$ \\
\hline $\mathrm{Eucl}_{F}$ & $\begin{array}{l}\text { Euclidean distance in between the best } \\
\text { forwarding node and the source node }\end{array}$ \\
\hline $\mathcal{T}_{d j}$ & Residual deadline time \\
\hline$d_{i}^{j}$ & $\begin{array}{l}\text { Euclidean distance between the forwarding } \\
\text { node } i \text { and the nearest sink } j\end{array}$ \\
\hline $\mathrm{CNI}_{r}$ & Cumulative congestion degree \\
\hline$C N I_{N R T M e m}$ & $\begin{array}{l}\text { Minimum buffer available in NRT traffic } \\
\text { with FIFO based storage }\end{array}$ \\
\hline$C N I_{R T D M e m}$ & $\begin{array}{l}\text { Minimum buffer available in RTD traffic } \\
\text { with prioritized queuing-based storage }\end{array}$ \\
\hline CNI $_{\text {NRTMemMax }}$ & Maximum buffer capacity of NRT traffic \\
\hline$C N I_{R T D M a x}$ & Maximum buffer capacity of RTD traffic \\
\hline $\mathbb{N}$ & Total nodes in the network \\
\hline$C N I_{r i}$ & Cumulative congestion degree for $i$-th node \\
\hline$\eta$ & Dynamic link quality \\
\hline$\alpha$ & Weight parameter \\
\hline $\mathbb{N}_{r x}$ & Total number of the received packets \\
\hline $\mathbb{N}_{t x}$ & Total number of the transmitted packets \\
\hline $\mathbb{V}_{t}$ & Speed factor \\
\hline $\mathbb{D}_{E S D}^{i}$ & Distance between source to destination \\
\hline $\mathbb{D}_{E N S}^{i}$ & $\begin{array}{l}\text { Distance between one hop neighbor node } \\
\text { to the destination }\end{array}$ \\
\hline$A R T T_{T i}$ & Average round trip time \\
\hline$C R M_{i}$ & Cumulative rank matrix \\
\hline$\omega_{1} / \omega_{2} / \omega_{3}$ & Weight parameters \\
\hline
\end{tabular}

The remaining sections of the paper are structured as follows. Section 2 discusses the related work, while the proposed routing model and its implementation are presented in Section 3. Section 4 discusses the results obtained and is followed by conclusions and future work recommendations. 
The list of abbreviations used in this paper is presented in Table 1.

\section{Related Work}

To achieve energy-efficient cooperative MIMO networks, Peron et al. [3] developed a cross-layer architecture where they applied PHY and MAC layers. In their proposed method, the authors estimated the outage probability based on power transmission estimation at PHY layer. In addition, at MAC layer, they examined different channels taking more time and energy, based on which they performed energy-efficient cooperative MIMO communication. Su et al. [4] developed a cross-layered cooperative transmission model which was applied in an interference channel in conjunction with cooperative interference between transmitters to achieve cell-edge throughput optimization. To assist efficient resource allocation in cooperative communication, they introduced an enhanced dirty paper coding at the PHY layer which rendered a better achievable rate region. Furthermore, they developed a cooperative transmission scheduling model at the MAC layer that enabled cooperative nature as per channel condition variation. Their model was found to be better in terms of throughput.

Rao et al. [5] performed optimum power allocation and resource management by exploiting the cross-layer model for throughput optimization in WSNs. Chen et al. [1] focused on ensuring reliable data transmission over WSNs, where they recommended a scalable, energy-efficient, and error-resilient routing model. To achieve the desired result, the authors developed a cross-layer model-based distributed energy-efficient and reliable routing protocol, where they amalgamated the network layer and power allocation policy at the PHY layer. To perform BFN selection, the authors considered the route with minimum power consumption and higher end-to-end reliability.

Mythrehee et al. [2] developed a cross-layered underwater wireless sensor networks (UWSNs) routing protocol that applied the adaptive neuro fuzzy-based interference system for measuring the depth of the sensor nodes, and the game theoretic model for localization of the sensor nodes at the upper layer of the sea.

Patil et al. [6] focused on throughput optimization of WSNs using the cross-layer routing model. The authors recommended maintaining a timely data delivery capacity in WSNs and, therefore, they developed an integrated cross-layer model. The cross-layer model they proposed focused primarily on memory allocation and power allocation functions for WSNs. Imen et al. [7] focused on energy-efficiency and increased life span of WSNs. The authors stated that the available zone routing protocols (ZRP) cannot deal with the adverse factors affecting the network, especially in large scale networks, and hence proposed a hierarchical cross-layer model based on the routing protocol (H-ZRP). Their model was found better in terms of packet loss rate and transmission rate.
Wan et al. [8] developed the QoE-oriented cross-layer resource allocation model for open wireless networks (OWN), with a mapping function applied between the service rate and the mean opinion score for best effort services. Ozen et al. [9] developed a two-tier SD and multi-rate transmission model for the cross-layered MAC design, which was used for QoS-centric communication over multimedia sensor networks (WMSNs). Wang et al. [10] developed an adaptive-opportunistic aloha (A-OAloha) for the UAVWSN system to support network efficiency. A-OAloha was in fact a cross-layer model developed for successful data transmission and energy optimization over WSNs.

Chen et al. [11] developed novel wireless networked control systems (WNCSs) for which a cross layer network design was suggested to achieve network awareness under critical real-time traffic variations. Their proposed cross-layer model adaptively adjusted the control period to achieve improved resource utilization while maintaining timely data delivery. Mezouary et al. [12] developed a cross-layer model-based SD scheme to classify data as RTD and NRT traffic in WSNs. They combined the parameters from the MAC layer and the network layer to augment throughput. Peng et al. [13] focused on balancing the trade-offs between different activities, such as energy consumption and packet collision, proposing a cross-layer routing model in conjunction with a directed spanning tree routing algorithm. The algorithm they proposed resolved the key issue of undesired energy exhaustion during the transmission.

Xiong et al. [14] developed a cross-layer architecturebased MAC optimization model for WSNs. The authors applied a special inference ruler for MAC by employing computational geometry methods. Mishra et al. [15] also recommended the cross-layer WSN design to achieve QoS in WSNs. Neela et al. [16] developed an adaptive cross-layer model to augment the functions of the different layers. In opposition to classic routing and MAC layer (RMC) protocols using clustering, they proposed Enhanced-RMC (E-RMC) to achieve a higher network lifetime. Singh et al. [17] developed a cross-layer contention-based synchronous MAC protocol for WSN with multi-hop transmission. Considering the limitations of multi-hop transmission, such as reduced PDR and higher end-to-end delay, the authors proposed a cross-layer contention-based synchronous MAC protocol that collects the request-to-send data process in the data window and the confirmation-to-send data process in the sleep window to increase efficiency.

Anugraha et al. [18] focused on augmenting cooperative relaying in interference-limited multi-hop networks to achieve multi-rate transmission and power control. They developed a cross-layer flow-based routing model that jointly augments the routing parameters for better scheduling. Peng et al. [19] focused on congestion avoidance in multi-hop transmission-based WSNs and proposed a crosslayer model-based information exchange over a cross-layer design established between MAC and routing layers. Yuan et al. [20] developed a multi-hop virtual multiple-input- 
multiple-output (VMIMO)-based cross-layer routing protocol design that augments energy efficiency, reliability and end-to-end QoS provision over WSNs. They achieved better energy exhaustion and an optimized set of transmissions. A similar effort was made by Shan et al. [21] who developed a QoE driven cross-layer resource allocation model for high traffic services over the OWN downlink. Similarly, the cross-layer model has been applied for ad-hoc and MANET purposes as well [22]-[29].

In [22], Gawas et al. developed a cross-layer model-based cooperative routing model over vehicle ad-hoc networks (VANETs). The authors developed the cross-layer model to ensure reliable data transmission for safety-related messages, with minimum end-to-end delay. Their model focused on achieving one-hop relay node, to ensure a reliable message broadcast. Rath et al. [23] proposed a QoSoriented cross-layer routing model using network layer information and relying on exchange with other layers. They derived the rate monotonic algorithm (RMA) and earliestdeadline-first (EDF) scheduling to achieve a low deadline miss rate.

Shafi et al. [24] developed a cross-layer design-based cooperative routing model over VANET to achieve higher throughput and low loss ratios. Unlike [22], Gawas et al. focused, in [25], on achieving multilayer functionality from the PHY layer to the routing layer, to accomplish cooperative communication over MANET. They developed an adaptive cross-layered cooperative routing algorithm (ACCR) which exploits channel state variations to select the cooperative MAC model by employing spatial diversity information. Similarly, in [26] they proposed the IEEE 802.11e enhanced distributed channel access (EDCA) routing protocol for QoS-centric multimedia transmission. They focused on MAC optimization and MULTI-metric link disjoint multi-path routing (CMMR). They used MAC queue utilization, node density degree, and mobility to achieve channel-state awareness and routing decision capabilities.

In [27], PHY and MAC layers were used to design a crosslayer routing model for VANET. Nithya et al. [28] developed a QoS-centric multi-hop ad-hoc routing protocol by amalgamating MAC layer contention resolution and TCP layer congestion control. To achieve congestion control, they the applied Fibonacci sequence. Elias et al. [29] developed a random network coding (RNC)-based routing model for cooperative communication over VANET. They proposed the RECMAC model to achieve higher transmission reliability and throughput values.

\section{Proposed CL-mWSN Protocol}

To ensure QoS and reliable data communication over mobile WSNs, optimized forwarding path selection is vital. Excessively high topological variations may occur, predominantly under dynamic topology conditions, which may force the network and the nodes to undergo severe transitions, and may result in network parameter changes. BFN selection is a highly intricate task under such conditions. While performing BFN selection under such conditions, maintaining efficient (network) information is a must, which may help in making optimized, proactive routing decisions. BFN plays an important role in QoS-centric and reliable transmission over mobile WSNs. To achieve it, we assume that each deployed node has information about nodes located at the distance of one-hop. Here, to deal with dynamic topology, a proactive node management and routing protocol has been proposed.

In the proposed routing protocol, each node possesses a routing protocol that assists it in obtaining node information from different layers of the protocol stack. With QoS objectives considered, the proposed model exploits dynamic link quality, congestion probability at the node, buffer availability, packet velocity, packet injection rate or velocity, etc. These parameters may be applied to examine the suitability of a given node to become BFN for reliable data transmission. On the other hand, selection of the BFN depends, primarily, on multiple parameters, such as buffer capacity, packet injection rate, link quality, etc. The proposed routing protocol exploits those parameters from the different layers and enables reliable data transmission. In addition, considering mission critical communication purposes, where enabling timely data delivery is of utmost significance a novel service differentiation and fair resource scheduling model is developed in this research paper. The proposed SD model is capable of classifying data as RTD and NRT - a feat that has been augmented further by a novel QoS-centric fair resource allocation strategy.

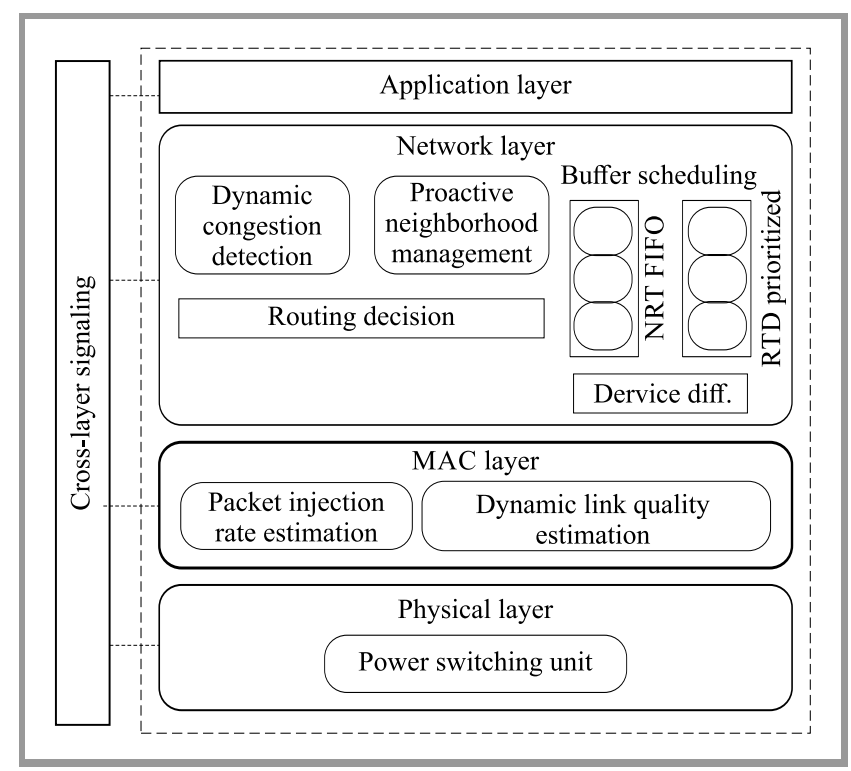

Fig. 1. Proposed cross-layer architecture-based QoS-centric routing protocol for mobile WSNs (CL-mWSN).

A snippet of the proposed cross-layer architecture is given in Fig. 1. CLmWSN exploits the network layer and the MAC layer of the IEEE 802.15.4 protocol stack. At the network layer, the CL-MWSN protocol is capable of service differentiation and fair resource scheduling (SDFRS), 
dynamic congestion detection (DCD) and proactive network/node table management. Similarly, at the MAC layer of the IEEE 802.15.4 standard, packet injection rate of velocity (per node) and dynamic link quality have been estimated.

As stated in Fig. 1, CL-mWSN exploits primarily the application, network and MAC layers of the IEEE 802.15.4 protocol stack. The CL-mWSN model applies different functions to different layers. The key functions of the CLmWSN routing protocol are:

- proactive network table management,

- service differentiation and fair resource allocation,

- congestion detection and avoidance model,

- dynamic link quality measurement,

- packet velocity measurement,

- cumulative rank matrix estimation and best forwarding path selection.

\subsection{Proactive Network Table Management}

Typically, nodes in classic mobile WSNs having their parameters, such as energy capacity, maximum buffer capacity, radio range, etc. defined, and due to the dynamic topology of the network, key parameters, such as link connectivity and buffer availability may change over time. Such changes may even be triggered by varying payload conditions, varying signal-to-noise ratio or link connectivity changes. In certain situations a node might may be forwarding data for a long time, which prevents data from reaching its destination within the defined time limit. Under varying topological conditions, a node may suffer from buffer deficiency and may require an additional buffer to ensure a reliable data transfer. Similarly, over the simulation period in mobile WSNs, the inter-node distance may vary and link connectivity may be subject to change as well. In such conditions, static or predefined parameter-based routing decisions can lead to link outages.

Due to considerable variations in network parameters, updating these parameters pro-actively is unavoidable. In other words, to cope with their dynamic topology, mobile WSNs require robust, proactive network management and a node table updating strategy. With this motivation in mind, the CL-mWSN protocol proposed in this paper relies on proactive network and node table management that enables dynamic network or/and node parameter updates to assist reliable BFN selection and routing decision process. It leaves out the continuous node discovery phase - an approach that reduces signaling overhead and energy consumption. In the proposed routing approach, node parameters are updated dynamically, which facilitates swift routing decision making. Each node maintains details of the singlehop neighboring node by transmitting a beacon message. The message comprises significant information about the node, along with its characteristics, such as NodeID, highest buffer capacity, current (available) buffer status, node position, packet holding period, current packet velocity, dynamic or current link quality, etc.

The three parameters: NodeID, node position and current link quality are communicated through the beacon message or ACK. This reduces computational cost and memory usage. Each control packet comprises 42 bytes and is split into three fields; NodeID (16 bits), current node status (192 bits to store link quality, current buffer availability) and node position field (128 bits). Each transmitting beacon message collects information on the on-hop neighboring node, which is updated continuously. One of the key issues in mobile WSNs is packet collision during transmission and, therefore, to avoid it, the node multicasts a beacon message that operates in coordination with an offset timer. In the proposed CL-mWSN routing protocol, the use of the offset timer is based on a normal homogeneous distribution approach. Once a request for packet transmission has been received, the node resets its off-set timer. Here, a node located at the one-hop distance updates the nearest destination in the table.

Let $\mathbb{N}_{j}$ be the one-hop distant neighbor and $B F N_{i}$ be the potential or most suitable forwarding node. The node table is updated using Eq. (1), where $E_{u c l}$ and $E u c l_{d}$ signify the Euclidean distance in between the best forwarding node and the source node to the nearest destination:

$$
\mathbb{N}_{\text {Table }}=\left\{B F N_{i} \in \mathbb{N}_{j} \mid E u c l_{d}-E_{u c l} \geq 0\right\}
$$

\subsection{Service Differentiation and Fair Resource Scheduling}

To ensure QoS-centric communication, data awareness and associated resource scheduling play a decisive role. There are numerous application environments where the provision of sufficient resources (i.e. buffer) for a successful or QoS-centric transmission is a must. In mobile WSNs different data types may be communicated, including RTD and NRT data. The provision of sufficient buffer or bandwidth for RTD data is a must. However, maintaining an optimized amount of resources for NRT data may be of utmost significance as well. In the CL-mWSN routing protocol, both RTD and NRT data have been considered. Here we assume that data could be classified into two broad types, RTD and NRT. Once the data has been identified, CL-mWSN intends to allocated resources to each data type, while maintaining an optimized amount of resources for RTD data and the maximum possible amount of resources for NRT data.

Each node is assigned with two distinct types of equal capacity buffers for RTD and NRT data. In the CL-mWSN model, where a node experiences a complete buffer exhaustion for RTD data and requires additional buffer capacity for a successful transmission, it may borrow the supplementary buffer capacity from the NRT buffer, where the data are stored in the normal FIFO manner. In mobile WSNs, both RTD and NRT buffers may be filled. In that 
case, CL-mWSN applies a fair resource scheduling approach (i.e. SDFRS), in which to meet the buffer-related demand for RTD data, NRT drops the recently added data to the FIFO queue. Although the data elements are stored in FIFO, the fact that a few recently connected elements are dropped cannot affect the overall performance in a significant manner. On the contrary, in major classic approaches, to provide additional buffer for RTD, the buffer for NRT is cleared or emptied completely, which is in violation of network QoS. Meanwhile, the proposed routing protocol enables optimal resource provision to RTD, while ensuring the maximum possible amount of resources for NRT, therefore balancing the resource utilization trade-offs to assure proper QoS. This mechanism allows to avoid long waiting times or holding periods at the, which ultimately augments overall network performance.

\subsection{Congestion Detection and Avoidance Model}

During a transmission over mobile WSNs, there is always the probability of a data flow that may as a result may impose congestion on a node. The probability of congestion increases considerably when we are dealing with mobile topology, which in turn increases the probability of a packet drop and retransmission, thus causing a QoS violation and energy exhaustion. To deal with this problem, the authors have recommended a timer-based transmission. In this mechanism, each node may transmit a beacon message to multiple nodes in the network whose frequency could be controlled through a predefined timer called the offset timer. Upon receiving a transmission request, CL-mWSN at first resets the associated timer, which eliminates ACK from the node. this also makes the proposed system computationally efficient and reduces signaling overheads. In addition, CL-mWSN avoids the storage of any significant paths or node-related information. Due to the dynamic topology, a node may receive more request to carry payload, exceeding its maximum carrying capacity, and buffer availability may vary over the simulations period, thus increasing the probability of congestion. This often results in a data drop and retransmission, causing energy exhaustion. To alleviate this problem, the CL-mWSN model implements a congestion detection and avoidance model (CDAM) that continuously assesses dynamic buffer capacity and the remaining buffer availability of node to detect congestion. It exploits the maximum buffer capacity of a node and the current buffer availability to assess the congestion probability at a node in a mobile WSN.

Upon transmitting the beacon message, the node may retrieve resource availability (i.e. buffer availability) of a onehop distant node. To ensure the provision of QoS and a reliable transmission, a node with sufficient buffer availability may be efficient enough.

Upon identifying a node whose buffer availability is lower than the memory expected or required for data transmission, CDAM avoids that node to assist the CL-mWSNbased BFN selection. Only a node with a sufficient buffer availability and with the congestion-free status is selected for BFN formation. This reduces any likelihood of a data drop, retransmission, end-to-end delay and energy consumption, which, in turn, augments QoS assurance in mobile WSNs.

In addition, the proposed CL-mWSN model functions in conjunction with the above stated SDFRS model that applies two distinct buffers for RTD and NRT data for each node, which fosters better resource management and allows to avoid congestion. As already discussed, in the SDFRS model, RTD data is stored in a prioritized manner, while NRT data is stored based on the FIFO methodology. As each packet is assigned in a real time application, with a predefined deadline, it requires the data to reach it within that deadline to make an optimized decision. TheCL-mWSN model offers a higher priority to RTD data, while offering the maximum amount of resources to NRT data. In this model, to ensure delay the respective deadline time. To achieve this objective, CL-mWSN considers the distance between the source sensor node and the sink. Noticeably, to facilitate the highest possible priority for event-driven RTD delivery over mobile WSNs, it is inevitable to have the minimum value of $\mathcal{T}_{\text {Ratio }}$ :

$$
\mathcal{T}_{\text {Ratio }}=\frac{\mathcal{T}_{d\lrcorner i}}{d_{i}^{j}}
$$

where $\mathcal{T}_{d i}$ signifies the residual deadline time, while $d_{i}^{j}$ states the Euclidean distance between the forwarding node $i$ and the nearest sink $j$. The deadline time is estimated by using the arrival time of the individual packet. $\mathcal{T}_{d_{j}}$ is updated for each packets before transmitting, and the queue time is subtracted from $\mathcal{T}_{d_{i}}$. Here, we use current buffer availability information to estimate the congestion probability at a mobile WSN node. In addition, the CL-mWSN model introduces a parameter called node congestion index (NCI), which comprises node information along with its association with a neighboring node subset $\mathbb{S}_{n}$. We estimate NCI using Eq. (3), where $C N I_{N R T M e m}$ and $C N I_{R T D M e m}$ signify the memory available in the NRT-related normal FIFO queue, and the RTD-related buffer in the prioritized queue, correspondingly. $C N I_{R T D M a x}$ and $C N I_{N R T M e m M a x}$ signify the highest memory or buffer capacity of the RTD and the NRT data. Thus, the overall CNI for connecting nodes in $\mathbb{S}_{n}$ may be obtained as:

$$
C N I_{r}=\frac{C N I_{\text {NRTMem }}+C N I_{R T D M e m}}{C N I_{\text {NRTMemMax }}+C N I_{\text {RTDMax }}}+\sum_{i=1}^{\mathbb{N}} C N I_{r i}
$$

Estimating the memory or the buffer available at each node and the associated congestion probability, the routing model decides whether that node qualifies to become the forwarding node. A congestion-free node with sufficient buffer availability is considered to be used as a forwarding node or for path selection purposes. This assures reliable data transmission over mobile WSNs with the lowest possibility of data drops and overflows. In addition, such an approach avoids the problem of packet collision. 


\subsection{Link Quality Measurement}

To ensure the provision of QoS in mobile WSNs, one needs to assess the quality of the link between the resilient communication, RTD transmission is scheduled based on participating nodes dynamically, in order to characterize the suitability of a node for becoming a BFN. Mobility could result in topological changes and, hence, inter-node distance variations. Fixed radio range dynamics may cause link quality variations based on inter-node distance. In such conditions, assessing link quality dynamically to decide its suitability for selecting a reliable forwarding path may be vital. The CL-mWSN model applies a proficient dynamic link quality estimation model at the MAC layer of the IEEE 802.15.4 protocol stack. Details of the dynamic link quality estimation model can be found in [30]. The CL-mWSN model applies the current ratio of received packets to estimate link quality:

$$
\eta=\alpha \eta+(1-\alpha) \frac{\mathbb{N}_{r x}}{\mathbb{N}_{t x}} .
$$

In the above equation, $\eta$ represents dynamic link quality. The packet received ratio defines the efficiency of the communication link. The remaining parameters $\mathbb{N}_{r x}$ and $\mathbb{N}_{t x}$ represent the total number of received and transmitted packets, respectively. Here, $\alpha$ remains within the range of 0 to 1 .

\subsection{Packet Injection Rate Estimation}

Selecting a node and, hence, the path with the minimum holding period specific for each node may be vital to reduce latency or end-to-end delay that eventually determines the provision of QoS. In the CL-mWSN model, we estimate the packet injection rate or the holding period of a given node, i.e. the time over which the node withholds data before forwarding it. A node with a minimum holding period or a maximum packet velocity or injection rate is considered for BFN selection. Here, we have applied the packet delay parameter to estimate packet velocity at each node. In CL-mWSN, packet delay is applied to estimate the inter-node distance between neighboring nodes and the nearest destination. CL-mWSN applies Euclidean distance and relative distance, round trip time $\left(A R T T_{T i}\right)$, etc., to estimate packet velocity. The Euclidian distance is obtained between the source and the nearest destination, while the relative distance is obtained between the neighboring node and the nearest destination.

A speed factor $\mathbb{V}_{t}$ is obtained using:

$$
\mathbb{V}_{t}=\frac{\mathbb{D}_{E S D}^{i}-\mathbb{D}_{E N S}^{i}}{A R T T_{T i}} .
$$

Applying Eq. (5), we have estimated the packet velocity $\left(\mathbb{V}_{\text {packet }}\right)$, using Eq. (6). $\mathbb{V}_{\text {packet }}$ signifies the highest rate of data transmission at a given transmission power rating $(\mathbb{P} t x)$ :

$$
\mathbb{V}_{\text {packet }}=\frac{\mathbb{V}_{t}}{\mathbb{R}_{\text {MaxSpeed }}} .
$$

In Eq. (6), $D_{E S D}^{i}$ signifies the Euclidean distance between source $i$ and the destination node. $D_{E N S}^{i}$ represents the distance between the source and the (nearest) sink. $R_{\text {MaxSpeed }}$ denotes the maximum possible speed of radio signal in air. In CL-mWSN the speed of radio signal is assumed to be equal to the speed of light, and round trip time is estimated as the time difference in time between packet transmission and reception of the acknowledge (ACK) signal:

$$
\operatorname{ARTT}_{T i}=\frac{\sum_{i=0}^{N} R_{A t}^{i}-v_{P t}^{i}}{\mathcal{N}} .
$$

In Eq. (7), variables $R_{A t}^{i}$ and $v_{P t}^{i}$ signify the time of receiving ACK and of packet transmission, respectively. The $\mathcal{N}$ states the total packets transmitted. Thus, estimating the packet velocity for each node we have used it as a node specific parameter to decide its suitability to be a BFN or path.

\subsection{Cumulative Rank Matrix Estimation and Best Forwarding Path Formation}

Once the dynamic network parameters of the participating nodes, as referred to above, have been estimated, they were used to select BFN. for this purpose, we estimated a (node) rank parameter called cumulative rank matrix (CRM) Eq. (8). As already stated, CL-mWSN applies three key network parameters: congestion probability, dynamic link quality and packet injection rate or velocity, to select the best forwarding path. This is followed by best forwarding path formation and data transmission. The proposed CRM value is obtained using:

$$
C R M_{i}=\omega_{1} \eta_{i}+\omega_{2} C N I_{i}+\omega_{3} V_{\text {packet }_{i}}
$$

In the above equation, $\omega$ denotes the weight parameter which can be decided based on network preferences or based on a specific environment. CRM signifies the cumulative rank of node $i$. Noticeably, $\omega$ is assigned in such a manner that $\sum_{i=1}^{3} \omega_{i}=1$. The variable $\eta$ denotes the dynamic link quality.

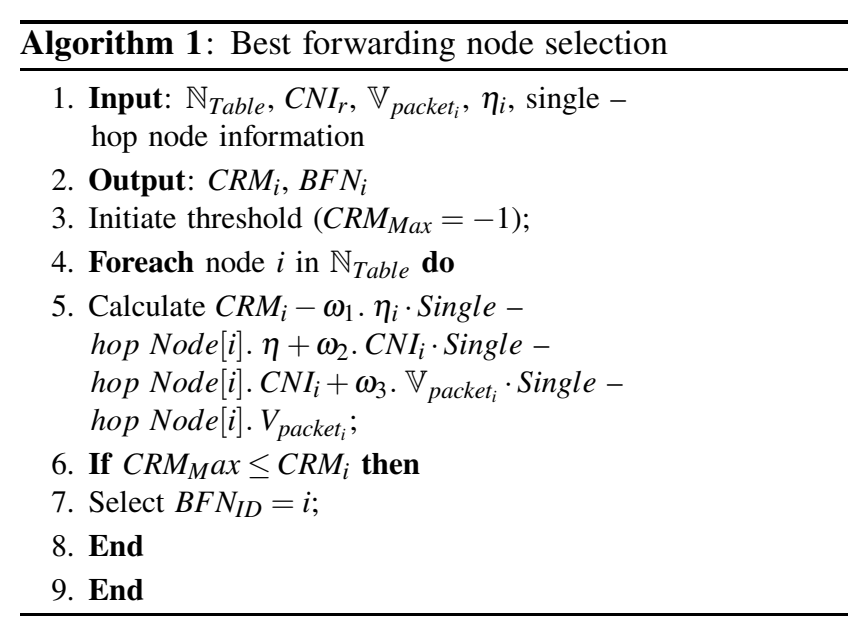


Table 2

CL-mWSN protocol parameters with their function

\begin{tabular}{|l|l|l|}
\hline 802.15.4 OSI layer & \multicolumn{1}{|c|}{ Parameter } & \multicolumn{1}{c|}{ Function } \\
\hline \hline \multirow{3}{*}{ Network layer } & Proactive node table - Eq. (1) & Proactive network table management \\
\cline { 2 - 3 } & Buffer availability-congestion - Eq. (3) & Congestion detection avoidance model \\
\cline { 2 - 3 } & Data type classification (RTD \& NRT) & Service differentiation and fair resource scheduling \\
\hline \multirow{2}{*}{ MAC layer } & Dynamic link quality - Eq. (4) & Link quality measurement \\
\cline { 2 - 3 } & Packet velocity - Eq. (6) & Packet rate injection estimation \\
\hline
\end{tabular}

Upon estimating CRM of each participating node, the estimated rank $C R M_{i \in \text { TotalNodes }}$ is updated in the decreasing order, and a node with the maximum CRN is considered as BFN for further data transmission over mobile WSNs. Algorithm 1 for BFN selection is then prepared for simulation purposes.

\section{Results and Discussion}

To examine the efficiency of the proposed CL-mWSN, we have compared its performance with other existing stateof-the-art cross-layer architecture-based routing protocols considering the parameters shown in Table 2 .

The majority of the existing approaches rely on single parameter-based routing decision-making schemes, where the emphasis is placed on augmenting PHY and MAC [3], [4], [9], [12], SD functions [9], congestion avoidance at MAC [19] etc. However, very few efforts have been made to make the routing decision while considering the type of data and its priority, to ensure deadline sensitive communication [12]. On the contrary, it is a fact that inclusion of these all factors, network condition-aware routing with synchronized cross-layer information may improve BFN selection and routing decisions.

With this motivation, in this research a reference model has been developed that combines the major efficacies of multiple contributions made, such as in [3], [4], [9], [12], [19], [22]. Although in [3], the authors considered only the MAC and PHY layers where throughput and link-outage probability (at MAC) were used to perform PHY switching control, they could not address other aspects, such as packet transmission rate or packet velocity of a node, which is a must for deadline-sensitive, mission-critical communication. Similarly, in [9], the focus was on employing the SD model to achieve multi-rate transmission for data-specific transmission; however, they could not address such issues those referred to in [3], or even in our work. In addition, they applied a classic prioritization scheme, which offered less or even no concern to fair resource scheduling for NRT data. Even the existing cross-layer protocols have failed to assess whether and how (i.e. up to what extent) their proposed resource scheduling affects QoS-centric delivery of NRT data. Similarly, authors in [22] developed crosslayer based cooperative routing model, where they focused on achieving a minimum delay. Therefore, they estimated the one-hop relay node to ensure reliable message broad- cast over VANET. They also applied MAC and transport layers to perform adaptive transmission rate control. However, they could not deal with other adverse effect caused by mobility. The work done in [31] looks better, as the have tried to implement multiple network parameters (of static WSN), such as velocity, energy to perform one-hop relay node identification or selection. This model could not offer the provision of better SD. Noticeably, the aforementioned routing cross-layer models [3], [4], [9], [12], [19] have implemented a cross-layer model, but for static WSNs only. They have not addressed the exceedingly dynamic topology of a mobile WSN. Under such conditions, development of a suitable reference model based on certain existing works referenced in [3], [4], [9], [12], [19], [22], [30] is inevitable, as it may help in assessing proposed model by applying common performance characteristics. With this motivation, a reference model, similar to the method suggested in [30], has been designed, amalgamating almost all features of or intentions referred to in [3], [4], [9], [12], [19]. Thus, the reference model [31], hereinafter re-

Table 3

Experimental setup

\begin{tabular}{|l|c|}
\hline \multicolumn{1}{|c|}{ Parameter } & Specification \\
\hline $\begin{array}{l}\text { Operating } \\
\text { system }\end{array}$ & $\begin{array}{c}\text { Windows 2010, 8 GB RAM, } \\
\text { Intel Core i5 processor }\end{array}$ \\
\hline Simulation tool & Matlab 2012b \\
\hline Protocol & CL-mWSN \\
\hline Data link & CSMA \\
\hline Physical & IEEE 802.15 .4 PHY \\
\hline MAC & $100 \mathrm{~m}$ \\
\hline Mobile nodes & $10 \mathrm{~s}$ \\
\hline Radio range & Circular \\
\hline $\begin{array}{l}\text { Packet } \\
\text { deadline time }\end{array}$ & \begin{tabular}{c} 
IEEE MAC \\
\hline Mobility
\end{tabular} \\
\hline $\begin{array}{l}\text { Weight } \\
\text { parameters }\end{array}$ & $480 \mathrm{~s}$ \\
\hline $\begin{array}{l}\text { Simulation } \\
\text { period }\end{array}$ & \begin{tabular}{c}
$\omega_{3(\text { P_Vel })}=0.3$, \\
\hline Payload
\end{tabular} \\
\hline
\end{tabular}


ferred to as the "existing system", combines the efficacies of other models and implements the SD model, packet velocity etc. to perform RTD and NRT data delivery. The performance comparison has been done for both CL-mWSN and existing systems [31]. Before discussing performance results obtained, the key parameters used in the experimental setup are presented in Table 3.

As depicted in Fig. 2, the CL-mWSN protocol exhibits higher PDR (98.4\%) for RTD - a result that is higher than in the existing model [31]. The existing cross-layer model, say real-time power aware routing protocol (RPAR), has exhibited a PDR of $93.1 \%$. Therefore, CL-mWSN has exhibited approximately $5.3 \%$ more PDR than the existing routing protocol.

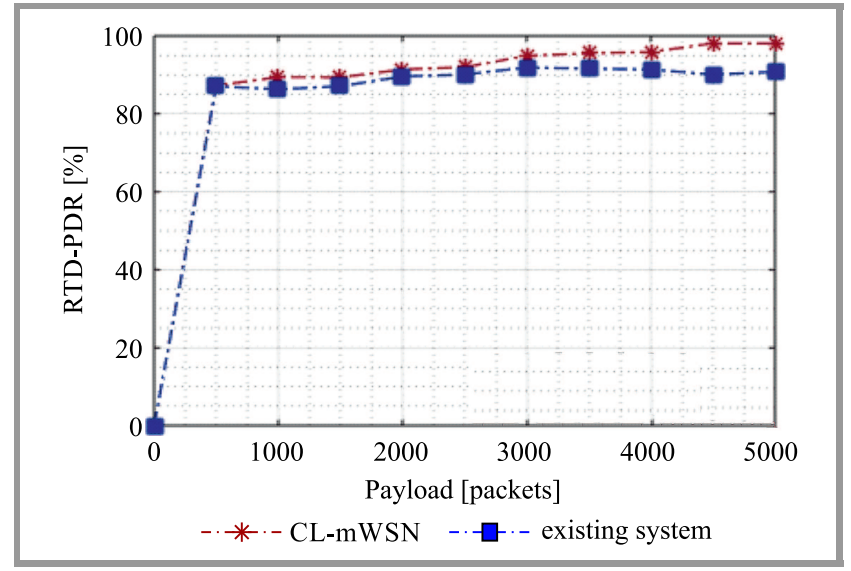

Fig. 2. PDR performance for RTD data.

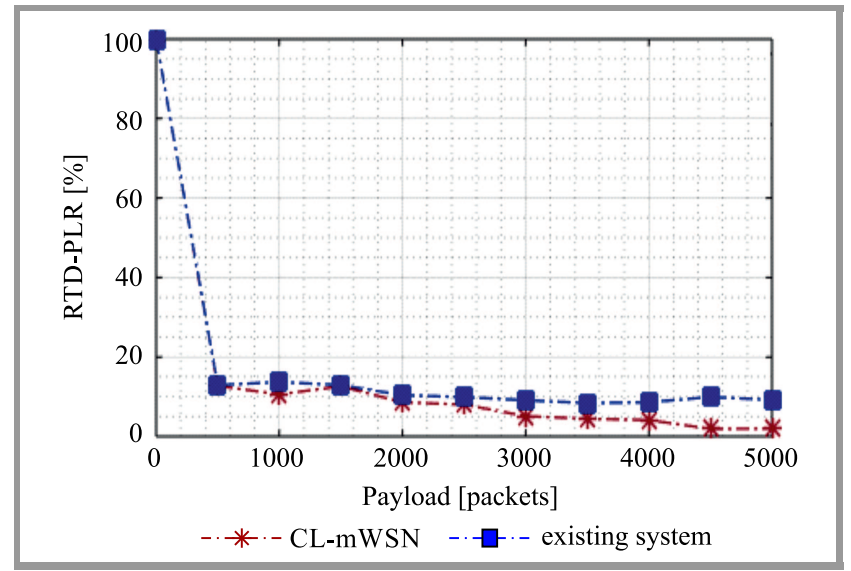

Fig. 3. PLR performance for RTD data.

As far as NRT-related performance is concerned, Fig. 3 shows the PDR value of the proposed routing model (93.8\%). This result reveals that CL-mWSN exhibits higher PDR while ensuring the maximum possible resource allocation and, hence, preserving QoS for NRT transmission. This signifies the robustness of the proposed routing model. Noticeably, even in mobile scenarios, PDR performance (98.4\%) for RTD traffic over mobile WSNs confirms robustness of the proposed routing model. Such a result allows to avoid the probability of retransmission and, hence, ensures proper QoS with minimum bandwidth utilization and transmission-related energy consumption.

PLR performance for RTD traffic typical of our proposed CL-mWSN model is depicted in Fig. 3. The CL-mWSN protocol exhibits a lower PLR (1.6\%) than the existing cross-layer routing protocol, RPAR $(6.9 \%)$. This efficacy backs up the robustness introduced in the proposed routing model. Unlike existing routing protocols, CL-mWSN employs different and dynamic network parameters to perform BFN or neighbor relay node selection, which eventually allows it to exhibit higher QoS provision levels. The higher throughput or PDR performance by CL-mWSN results in more successful data delivery and, hence, alleviates the probability of retransmission, which makes it energy efficient as well as resource efficient. The use SDRCRF makes CL-mWSN efficient and robust enough to ensure higher PDR for RTD, and guarantees uncompromised performance for NRT traffic.

PDR performance for NRT traffic is shown in Fig. 4. PLR for NRT traffic is presented in Fig. 5, where CL-mWSN has outperformed RPAR. CL-mWSN has exhibited PLR of merely $6.2 \%$ for NRT traffic.

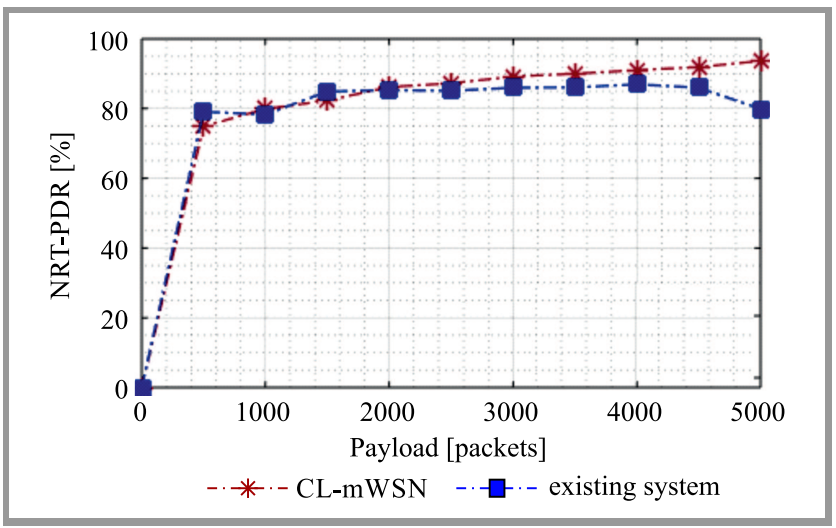

Fig. 4. PDR performance for NRT data.

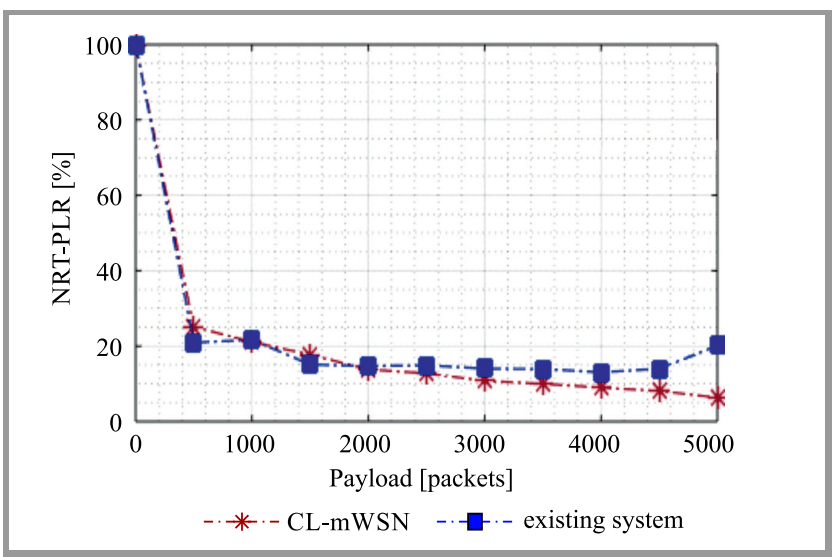

Fig. 5. PLR performance for NRT data.

The use of the novel SD model has strengthened CLmWSN ensuring optimized resource allocation for RTD traffic, while maintaining the maximum possible resources for NRT traffic under congestion and resource-constrained 
conditions. FIFO-based scheduling for NRT and last packet drop for accommodating RTD traffic are the parameters that allow CL-mWSN to exhibit a minimum drop of NRT data. This makes the proposed system robust for both RTD and NRT traffic transmissions.

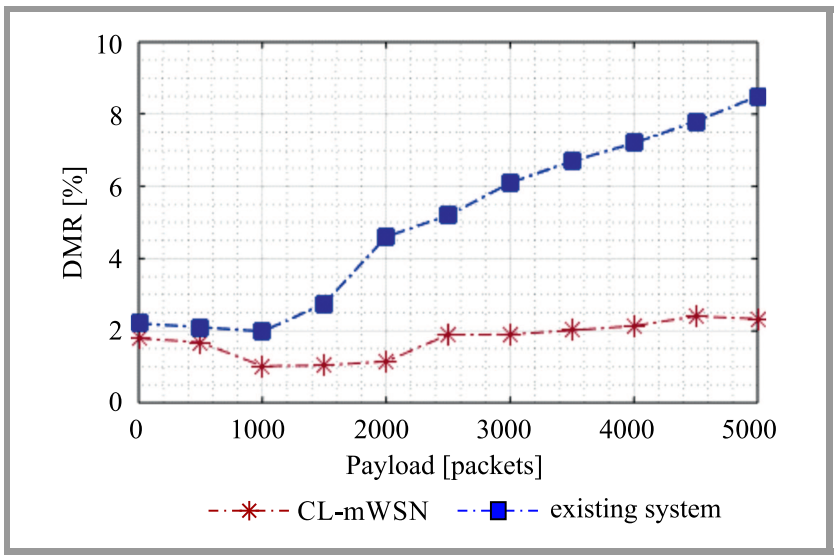

Fig. 6. DMR performance of routing protocol.

DMR performance is depicted in Fig. 6. Based on the results obtained, one may easily visualize that CL-mWSN exhibits lower DMR than the existing routing models [30]. This can be the result of deadline time (per packet) based data prioritization and resource allocation. Now, taking into consideration of all results discussed above and their respective significance, one may conclude that CL-mWSN performs better than other routing models, with its efficacy being relevant for used in real-time mobile WSN applications.

\section{Conclusion}

It may be stated that the proposed routing model offers a number of contributions, such as enhanced service differentiation and fair resource scheduling for different traffic types, while ensuring optimal PDR for RTD with allocating the maximum possible amount of resources to NRT. This allows the proposed system to achieve an optimized tradeoff for RTD and NRT transmissions over WSNs. Similarly, the inclusion of packet velocity of each node, describing the rate at which a given node may transmit data, has helped CL-mWSN ensure timely data delivery. Congestion avoidance and selection of the best forwarding note based on dynamic link quality also enabled the proposed routing protocol to achieve a maximum packet delivery ratio, a minimum packet loss ratio and, hence, a minimum retransmission probability, which eventually makes it energy-efficient. Therefore, the overall performance of the proposed routing protocol makes it robust enough to satisfy QoS demands in mobile WSN communication systems, which may be great significance for the IoT ecosystem as well. Although the proposed system attempts to add the greatest value to the routing model, it could not address the issue of dynamic power management and multi-rate switching control, which could have resulted in ensuring more bandwidth-efficient routing and in achieving higher energy efficiency.

In the future, efforts need to be made to incorporate the cross-layer design model with QoS-centric PHY switching or dynamic power management, in order to increase energy and resource efficiency.

\section{References}

[1] Y. Chen, L. Gao, Y. Xing, and W. Yi, "Cross-layer design for energyefficient reliable routing in wireless sensor networks", in Proc. 11th Int. Conf. on Mob. Ad-hoc and Sensor Netw. MSN 2015, Shenzhen, China, 2015, pp. 31-36 (doi: 10.1109/MSN.2015.44).

[2] H. Mythrehee and A. Julian, "A cross layer UWSN architecture for marine environment monitoring", in Proc. Global Conf. on Commun. Technol. GCCT 2015, Thuckalay, India, 2015, pp. 211-216 (doi: 10.1109/GCCT.2015.7342654).

[3] G. Peron, G. Brante, R. D. Souza, and M. E. Pellenz, "Physical and MAC cross-layer analysis of energy-efficient cooperative MIMO networks", IEEE Trans. on Commun., vol. 6, no. 5, pp. 1940-1954, 2018, (doi: 10.1109/TCOMM.2018.2796601).

[4] G. Su, B. Cui, and X. Wang, "Cross-layer approach to joint transmitter selection for cooperative transmission", in Proc. 16th Int. Symp. on Commun. and Inform. Technol. ISCIT 2016, Qingdao, China, 2016, pp. 426-430 (doi: 10.1109/ISCIT.2016.7751666).

[5] K. P. Rao and P. V. Sridevi, "An integrated cross layer approach for throughput improvement in wireless sensor networks", in Proc. 10th Int. Conf. on Intell. Syst. and Control ISCO 2016, Coimbatore, India, 2016, pp. 1-6 (doi: 10.1109/ISCO.2016.7727114).

[6] A. K. Patil and A. J. Patil, "Integrated cross layer controlling for wireless sensor network", in Proc. Int. Conf. on Pervasive Comput. ICPC 2015, Pune, India, 2015, pp. 1-6 (doi: 10.1109./PERVASIVE.2015.7087160).

[7] B. Imen and M. Abdellaoui, "Hierarchical organization by crossing between different layers for WSN energy saving", in Proc. 15th Int. Conf. on Sci. and Techniq. of Autom. Control and Comp. Engin. STA 2014, Hammamet, Tunisia, 2014, pp. 1020-1023 (doi: 10.1109/STA.2014.7086772).

[8] M. Wan, Z. Lu, L. Wang, X. Xia, and X. Wen, "A QoE-oriented cross-layer resource allocation scheme for mobile service over Open Wireless Network", in Proc. Int. Symp. on Wirel. Pers. Multim. Commun. WPMC 2014, Sydney, NSW, Australia, 2014, pp. 186-191 (doi: 10.1109/WPMC.2014.7014814).

[9] Y. Ozen, C. Bayilmis, N. Bandirmali, and I. Erturk, "Two tiered service differentiation and data rate adjustment scheme for WMSNs cross layer MAC", in Proc. 11th Int. Conf. on Electron., Comp. and Comput. ICECCO 2014, Abuja, Nigeria, 2014 (doi: 10.1109/ICECCO.2014.6997561).

[10] H. Li, L. Wang, S. Pang, and M. Towhidnejad, "A cross-layer design for data collecting of the UAV-wireless sensor network system", in Proc. 12th IEEE Int. Conf. on Embedded and Ubiquit. Comput., Milano, Italy, 2014, pp. 242-249 (doi: 10.1109/EUC.2014.43).

[11] Y. L. Chen, G. Tian, J. Gao, and Y. C. Tian, "Cross-layer design for traffic management in wireless networked control systems", in Proc. 9th IEEE Conf. on Indust. Electron. and Appl., Hangzhou, China, 2014, pp. 187-192 (doi: 10.1109/ICIEA.2014.6931156).

[12] R. El Mezouary, A. Loutfi, and M. El Koutbi, "A cross-layer architecture for service differentiation in wireless sensor networks with multiple sinks", in Proc. Int. Conf. on Multim. Comput. and Syst. ICMCS 2014, Marrakech, Morocco, 2014, pp. 843-848 (doi: 10.1109/ICMCS.2014.6911279).

[13] J. Peng, J. Jingqi, S. Qiushuo, and Z. Songyang, "A noble cross-layer protocol for QoS optimization in wireless sensor networks", in Proc. 26th Chinese Control and Decision Conf. CCDC 2014, Changsha, China, 2014, pp. 2430-2434 (doi: 10.1109/CCDC.2014.6852581).

[14] Q. Xiong and X. Li, "Cross-layer design of MAC and application semantics in wireless sensor networks", in Proc. 4th Int. Conf. on Commun. Syst. and Netw. Technol., Bhopal, India, 2014, pp. 147-150 (doi: 10.1109/CSNT.2014.38). 
[15] M. Mishra, G. S. Gupta, and X. Gui, "A review of and a proposal for cross-layer design for efficient routing and secure data aggregation over WSN", in Proc. 3rd Int. Conf. on Computat. Intell. and Netw. CINE 2017, Odisha, India, 2017, pp. 120-125 (doi: 10.1109/CINE.2017.30).

[16] N. Neela and O. B. V. Ramanaiah, "A comprehensive cross-layer framework for optimization of correlated data gathering in wireless sensor networks", in Proc. IEEE 6th Int. Conf. on Adv. Comput. IACC 2016, Bhimavaram, India, 2016, pp. 582-587 (doi: 10.1109/IACC.2016.113).

[17] R. Singh, B. K. Rai, and S. K. Bose, "A low delay cross-layer contention based synchronous MAC protocol for a multi-hop WSN", in Proc. IEEE Region 10 Conf. TENCON 2016, Singapore, 2016, pp. 1821-1824 (doi: 10.1109/TENCON.2016.7848335).

[18] M. Anugraha, A. Anitha, and J. J. Kumari, "Throughput optimization using cross layer flow-based framework in cooperative wireless multihop networks", in Proc. Global Conf. on Commun. Technol. GCCT 2015, Thuckalay, India, 2015, pp. 366-370 (doi: 10.1109/GCCT.2015.7342685).

[19] Q. Peng et al., "Multipath routing protocol based on congestion control mechanism implemented by cross-layer design concept for WSN", in Proc. IEEE 17th Int. Conf. on Computat. Sci. and Engin., Chengdu, China, 2014, pp. 378-384 (doi: 10.1109/CSE.2014.98).

[20] Yong Yuan, Zhihai He, and Min Chen, "Virtual MIMO-based crosslayer design for wireless sensor networks", IEEE Trans. on Veh. Technol., vol. 55, no. 3, pp. 856-864, 2006 (doi: 10.1109/TVT.2006.873837).

[21] L. Shan, Q. Liao, Q. Hu, S. Jiang, and J. Zhao, "A QoE-driven cross-layer resource allocation scheme for high traffic services over open wireless network downlink", in Proc. IEEE 82nd Veh. Technol. Conf. VTC2015-Fall 2015, Boston, MA, USA, 2015 (doi: 10.1109.VTCFall.2015.7390811).

[22] M. A. Gawas, L. J. Gudino, and K. R. Anupama, "Cross layer approach for effective multi hop broadcast in VANET", in Proc. 9th Int. Conf. on Commun. Syst. and Netw. COMSNETS 2017, Bangalore, India, 2017, pp. 403-404 (doi: 10.1109/COMSNETS.2017.7945414).

[23] M. Rath, B. Pati, and B. K. Pattanayak, "Cross layer based QoS platform for multimedia transmission in MANET", in Proc. 11th Int. Conf. on Intell. Syst. and Control ISCO 2017, Coimbatore, India, 2017, pp. 402-407 (doi: 10.1109/ISCO.2017.7856026).

[24] S. Shafi, B. N. Bhandari, and D. V. Ratnam, "An improved cross layer cooperative routing for vehicular networks", in Proc. Int. Conf. on Res. Adv. in Integr. Navig. Syst. RAINS 2016, Bangalore, India, 2016 (doi: 10.1109/RAINS.2016.7764427).

[25] M. A. Gawas, L. J. Gudino, and K. R. Anupama, "Cross layered adaptive cooperative routing mode in mobile ad hoc networks", in Proc. 22nd Asia-Pacific Conf. on Commun. APCC 2016, Yogyakarta, Indonesia, 2016, pp. 462-469 (doi: 10.1109/APCC.2016.7581425).

[26] M. A. Gawas, L. J. Gudino, and K. R. Anupama, "Cross layer multi QoS metric routing for multimedia traffic in $802.11 \mathrm{E}$ over MANETs", in Proc. 8th Int. Conf. on Ubiquit. and Fut. Netw. ICUFN 2016, Vienna, Austria, 2016, pp. 582-587 (doi: 10.1109/ICUFN.2016.7537099).

[27] S. Rehman, M. A. Khan, and T. A. Zia, "Cross layer routing for VANETs", in Proc. of IEEE Int. Symp. on a World of Wirel., Mob. and Multim. Netw. 2014, Sydney, NSW, Australia, 2014 (doi: 10.1109/WoWMoM.2014.6919006).

[28] B. Nithya, C. Mala, and E. Sivasankar, "A novel cross layer approach to enhance QoS performance in multihop ad-hoc networks", in Proc. 17th Int. Conf. on Netw.-Based Inform. Syst., Salerno, Italy, 2014, pp. 229-236 (doi: 10.1109/NBiS.2014.13).

[29] E. C. Elias, S. Zhang, E. Liu, E. N. Nweso, and E. C. Joy, "RECMAC: Reliable and efficient cooperative cross-layer MAC scheme for vehicular communication based on random network coding technique", in Proc. 22nd Int. Conf. on Autom. and Comput. ICAC 2016, Colchester, UK, 2016, pp. 342-347 (doi: 10.1109/IConAC.2016.7604943).

[30] O. Chipara et al., "Real-time power-aware routing in sensor networks", in Proc. 14th IEEE Int. Worksh. on Qual. of Serv., New Haven, CT, USA, 2006 (doi: 10.1109/IWQOS.2006.250454).
[31] A. Woo and D. Culler, "Evaluation of efficient link reliability estimators for low-power wireless networks", Tech. Rep. UCB/CSD-031270, EECS Department, University of California, Berkeley, 2003 [Online]. Available: https://www2.eecs.berkeley.edu/Pubs/ TechRpts/2003/CSD-03-1270.pdf

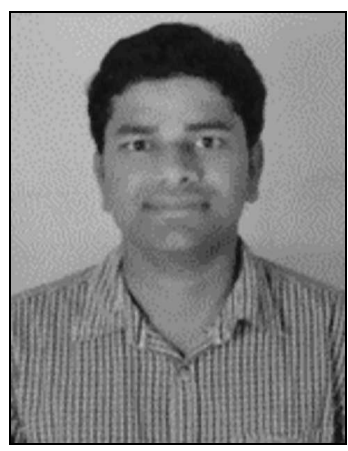

Kummathi Chenna Reddy is working as a Research Scholar at the Department of Electronics and Communication Engineering at REVA University. He received his M.E. and B.Tech. degrees in Electronics and Communication Engineering. His research interests include wireless sensor networks and neural networks.

Email: chenna.cr@gmail.com

School of Electronics \& Communication

REVA University

Bangalore, India

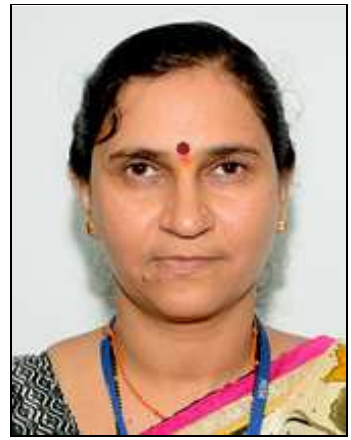

Geetha D. Devanagavi received her Ph.D. M.Tech. and B.E. degrees in 2014, 2005, and 1993, respectively. She is currently working as a Professor at Reva University. She has 21 years of teaching experience. Her research interests include wireless sensor networks, network security, and computer networks.

Email: dgeetha@reva.edu.in

School of Electronics \& Communication

REVA University

Bangalore, India

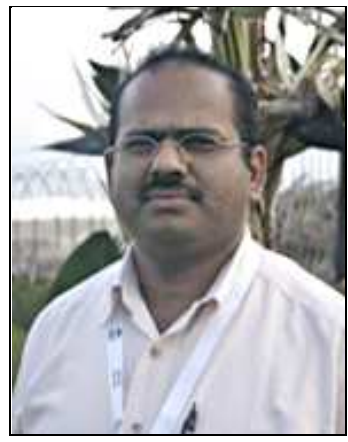

Thippeswamy M. N. is currently a Professor at CSE, NMIT, Bangalore, holds a B.E. degree in Computer Science and Engineering from Kuvempu University, India, M.Tech. degree in Computer Science and Engineering from VTU, India and Ph.D. in Engineering from the School of Engineering (Electrical, Electronic and Computer Engineering), Howard college campus, University of KwaZulu-Natal, Durban, South Africa. His interests focus currently on the Internet of Things, big data analytics, wireless ad hoc \& sensor networks and cognitive radio, with a particular emphasis placed on design and analysis of MAC and routing protocols.

Email: thippeswamymn@nmit.ac.in

Department of Computer Science

Nitte Meenakshi Institute of Technology

Bangalore, India. 